\title{
Formation of a Polyelectrolyte Complex from Carboxymethyl Cellulose and Poly(ethylenimine)
}

\author{
Hiroko Sato and Akio Nakajima \\ Department of Polymer Chemistry, Kyoto University, Kyoto 606, Japan.
}

(Received October 4, 1974)

\begin{abstract}
Formation of a polyelectrolyte complex was investigated as a function of $\mathrm{pH}$ by using carboxymethyl cellulose and poly(ethylenimine) as polyanion and polycation components, respectively. Experimental data on turbidity and conductometric and potentiometric titrations led to the conclusion that the formation of the polyelectrolyte complex did not obey stoichiometry. Such a result may be attributed to the less flexible nature of the polyanion molecular chains and to the distribution of ionizable groups on the chains of both components.
\end{abstract}

KEY WORDS Polyelectrolyte Complex / Stoichiometry / Carboxymethyl Cellulose / Poly(ethylenimine) / Turbidity /

When a polyelectrolyte reacts with an oppositely charged polyelectrolyte in an aqueous solution, a polyelectrolyte complex is formed. The complex formation with polyelectrolytes may differ from salt formation with microelectrolytes from the viewpoint of molecular conformations, i.e., the formation of a polyelectrolyte complex may not necessarily always be stoichiometric, if, for example, the chain are rigid. The reaction to form a polyelectrolyte complex is influenced by the degree of polymerization, the ionic strength of the medium, the reaction temperature, etc., and depends on $\mathrm{pH}$ if at least either the polyanion or the polycation is a weak polyelectrolyte. The polyelectrolyte complex is obtained in the state of a precipitate, a gel or a sol, depending on conditions. In a previous paper, ${ }^{1}$ we have reported a thermodynamic investigation on the complex coacervation phenomena, a phase separation in which polyelectrolytes participate, by using a polycation and a polyanion, both prepared from poly(vinyl alcohol).

Backbone chains of vinyl polymers, for example, are regarded to be flexible, while chains of polysaccharides and of polypeptides under some particular conditions are considered to be rigid. The chain flexibility of component polymers should affect the formation and the structure of the complex.

In most papers reported to data, complex formations performed under a stoichiometric relation have been investigated. The colloid titration ${ }^{2,3}$ is performed under such a conditions. Michaels $^{4}$ has pointed out that the reaction of two strong polyelectrolytes with opposite charges, sodium poly(styrene sulfonate) and poly(vinylbenzyl trimethyl ammonium chloride), was stoichiometric. Hosono ${ }^{5}$ examined the complex formation from partially sulfated poly(vinyl alcohol) and poly(vinyl alcohol) partially acetallyzed with diethoxy ethyl trimethyl ammonium salt, both of which are strong polyelectrolyte, and has pointed out that the reaction was stoichiometric and did not depend on the $\mathrm{pH}$ of the medium.

With respect to complexes made by reaction of a strong polyelectrolyte with a weak polyelectrolyte, Fuoss, et al., ${ }^{6}$ showed that poly(4vinyl- $n$ - $N$-butyl pyridonium bromide) (strong polybase) reacted with sodium polyacrylate (weak polyacid) in nearly stoichiometrical equivalence, and Hosono, et al., ${ }^{7}$ have pointed out that partially sulfated poly(vinyl alcohol) reacted with partially aminoacetalyzed poly(vinyl alcohol) (weak polybase) stoichiometrically as a function of the degree of dissociation of the weak polyelectrolyte.

The aim of this work is to elucidate the effect of chain flexibility on the stoichiometry of interactions, i.e., the equivalence of ammonium $\left(\mathrm{N}^{+}\right)$in polyethylenimine (PEI) and carboxylate $\left(\mathrm{COO}^{-}\right)$in carboxymethyl cellulose (CMC), as a function of $\mathrm{pH}$. Both of these are weak poly- 
electrolytes. The PEI molecule is a branched and flexible chain and its density of ionizable groups is high, while the CMC molecule has a rather rigid backbone chain.

\section{EXPERIMENTAL}

\section{Materials}

CMC having the degree of substitution of 0.75 was supplied by Daiichi Kogyo Seiyaku Co. The material was deionized by being passed through a column in which a mixture of anionand cation-exchange resins was packed. PEI provided by Nippon Shokubaikagaku Kogyo Co., was deionized by the same method as CMC after dialysis in pure water. The molecular weight of PEI was estimated as 9000 from viscosity data. ${ }^{8}$ Figure 1 shows a conductometric titration curve for PEI obtained without taking into account the effect of dilution. According to the statement of the manufacturer, the approximate ratio of primary, secondary, and tertiary amines in this PEI was said to be $1: 2: 1$. The straight line portion $a b$ in Figure 1 corresponds to the titration of the primary amine, and bc may be attributed to the secondary and tertiary amines. The experimental result shown in this figure

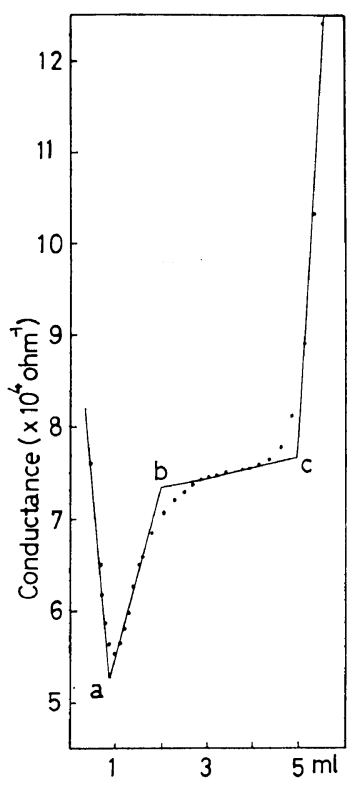

Figure 1. Conductometric titration of PEI aqueous solution with $0.1-N \mathrm{HCl}$. thus leads to the conclusion that the ratio of primary amine to the sum of secondary and tertiary amines is approximately $1: 3$. More quantitative discussion is impossible from this curve, but the PEI used should have a certain number of branches.

\section{Apparatus}

Concentrations $C$ of polyelectrolyte aqueous solutions in units of equivalent $/ l$, normality, were measured with a Yanagimoto Conductivity Outfit Model MY-8 in a cell with platinum black electrodes. Conductivities of polyelectrolyte complex solutions were measured in a cell with platinum electrodes. A Yanagimoto $\mathrm{pH}$ meter Type 7 with a sensitivity of $0.005 \mathrm{pH}$ was used for $\mathrm{pH}$ measurements. Turbidities were measured at the wave length of $436 \mathrm{~nm}$ with a ShimadzuKotaki Photoelectric Nephelotitrator Type NT $3 \mathrm{H}$. A $6 \times 10^{-3} \%(w / v)$ aqueous solution containing an equivalent amount (the number of ionizable groups in CMC is equal to that of PEI) of CMC and PEI was used as a control solution. This control solution was opalescent, and stable for a month or more without precipitation. Turbidities of given polymer solutions were obtained as relative values $(\%)$ referred to the turbidity of the control solution.

\section{RESULTS AND DISCUSSIONS}

The degrees of dissociation, $\alpha_{\mathrm{CMC}}$ and $\alpha_{\mathrm{PEI}}$, of the carboxyl groups in CMC and of the ionizable groups in PEI, respectively, are expressed by

$$
\begin{aligned}
\alpha_{\mathrm{CMC}} & =\frac{[\mathrm{NaOH}]+\left[\mathrm{H}^{+}\right]-\left[\mathrm{OH}^{-}\right]}{C_{\mathrm{CMC}}} \\
\alpha_{\mathrm{PEI}} & =\frac{[\mathrm{HCl}]+\left[\mathrm{OH}^{-}\right]-\left[\mathrm{H}^{+}\right]}{C_{\mathrm{PEI}}}
\end{aligned}
$$

where $[\mathrm{NaOH}]$ and $[\mathrm{HCl}]$ are the normalities of titrants, sodium hydroxide, and hydrochloric acid, respectively, and $\left[\mathrm{H}^{+}\right]$and $\left[\mathrm{OH}^{-}\right]$are those of free hydrogen and hydroxyl ions, respectively, in aqueous solution at each $\mathrm{pH}$ in the absence of polyelectrolytes. The activities of these ions in polyelectrolyte solutions were assumed to be the same as those in aqueous solutions. In Figure 2, the degrees of dissociation of CMC and PEI calculated from eq 1 and 2 are plotted 


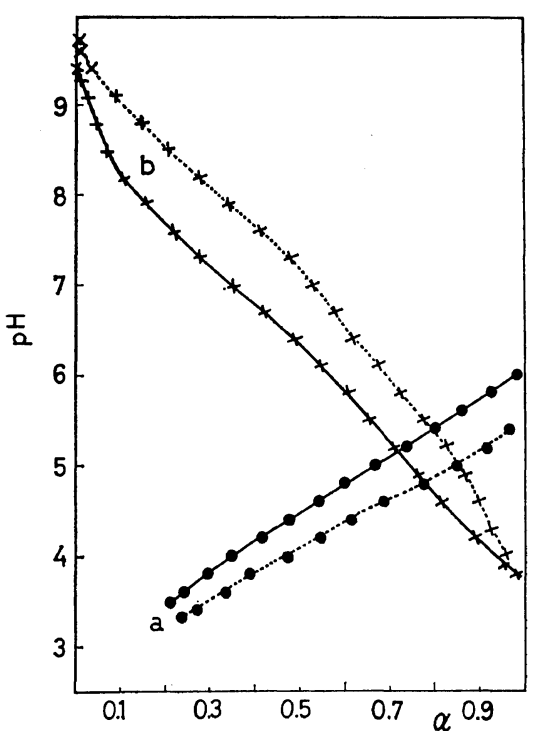

Figure 2. $\mathrm{pH}$ plotted against the degree of dissociation $\alpha$ for CMC (a) and PEI (b) in $\mathrm{H}_{2} \mathrm{O}$ (full line) and in 0.01- $M \mathrm{NaCl}$ solution (broken line) at $25 \pm 0.01^{\circ} \mathrm{C}$.

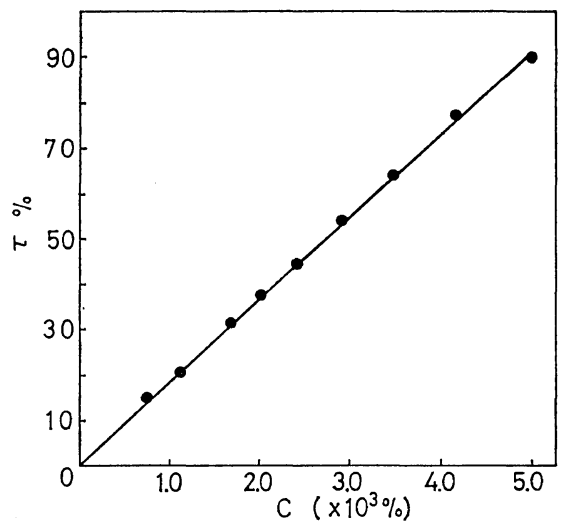

Figure 3. Dependence of turbidity on polymer concentration by successive dilution of a $5 \times 10^{-3} \%$ solution of $\mathrm{PEI} / \mathrm{CMC}$ equivalent mixtures.

against $\mathrm{pH}$ for systems in the absence and in the presence of $0.01-M \mathrm{NaCl}$. As is obvious from the figures, the effect of ionic strength on dissociation is rather large. Therefore, data for systems free from $\mathrm{NaCl}$ are used in the following discussion.

When a dilute aqueous of CMC was mixed with that of PEI, the solution immediately became turbid, and its turbidity reached a definite value in a few minutes. We observed no change in the turbidity even if the temperature of a solution in which the polyelectrolyte complex had already formed was raised from 18 to $45^{\circ} \mathrm{C}$. The effect of polymer concentration on the turbidity $\tau$ is shown in Figure 3. This figure shows that a linear relationship is set up between them when a complex solution of CMC and PEI $(1: 1)$ is diluted successively. Turbidities of complex solutions, measured for a mixed solution by adding an aqueous solution of PEI (or CMC) successively to that of CMC (or PEI), were calibrated to a proper polymer concentration, assuming that the turbidity is proportional to the polymer concentration.

The mixing ratio of PEI, $R_{\mathrm{PEI}}$, in the mixture of CMC and PEI is denoted by

$$
R_{\mathrm{PEI}}=\frac{n_{\mathrm{PEI}}}{n_{\mathrm{PEI}}+n_{\mathrm{CMC}}}
$$

where $n_{i}$ is the total number of ionizable groups of $i$ component in a mixture. In Figure 4, the relative turbidity is plotted against $R_{\mathrm{PEI}}$. The turbidity was sensitively affected by the concentration of solution, methods of mixing, ionic strength, etc., i.e., the shape of the turbidimetric curves was dependent on these factors. Further, any aggregation formed in the polyelectrolyte complex solution decreased the turbidity to some extent. However, the $R_{\mathrm{PEI}}$ at the maximum turbidity $\tau_{\max }$ is the same for either case, where

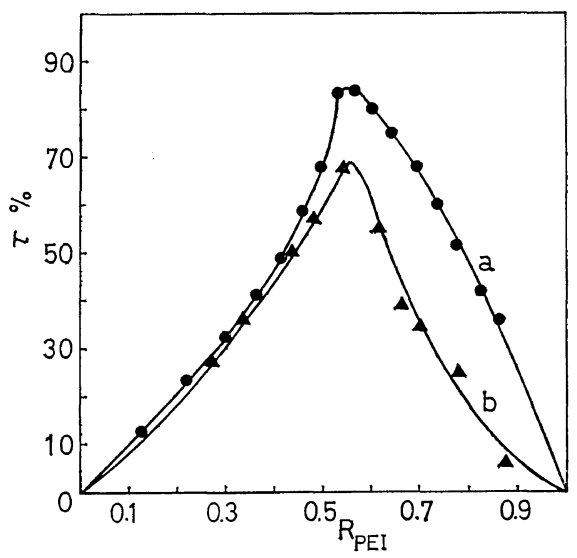

Figure 4. Relations between turbidity and mixing ratio $R_{\text {PEI }}$ obtained by two methods of mixing: a, addition of PEI solution into CMC solution; b, addition of CMC solution in PEI solution; polymer concn, $4.01 \times 10^{-3} \%$. 
the PEI solution is added to the CMC solution or where the CMC solution is added to the PEI solution, as is obvious from Figure 4.

The presence of a microsalt in a solution composed of polyanion and polycation suppressed ${ }^{1,5,9}$ the formation of polyelectrolyte complex when both components existed in equivalent amounts, but increased it in a certain concentration range of the microsalt when both components were not equivalent. Further, the presence of a microsalt may make the location of $\tau_{\max } v s$. mixing ratio obscure, and influences the degree of dissociation, as mentioned above. Accordingly, in this work, measurements of turbidity were carried out with systems involving no microsalt, Thus, a buffer solution was not used, and the $\mathrm{pH}$ 's of the CMC or PEI solution were adjusted to the desired values by adding $0.1-N$ $\mathrm{HCl}$ or $0.1-\mathrm{N} \mathrm{NaOH}$.

In Figure 5, the turbidity of the mixture is plotted against the $\mathrm{pH}$ of the mixture by adding a $6 \times 10^{-3}-\%(w / v)$ PEI solution to $155 \mathrm{ml}$ of $2 \times 10^{-3}-\%(w / v)$ CMC solution. We can point out that the curve near the maximum point is very sharp, i.e., the deviation of $\mathrm{pH}$ near the maximum point is very small.

Figure 6 shows the relation between the turbidity and the mixing ratio $R_{\mathrm{PEI}}$ at five different $\mathrm{pH}$ values. In this case the $\mathrm{pH}$ 's of both CMC and PEI solutions were adjusted to be approximately the same. However, the largest change of $\mathrm{pH}$ due to mixing of both polymer solutions was observed at $\tau_{\max }$. Table I shows the $\mathrm{pH}$ values of component solutions before mixing and that of the mixture at $\tau_{\max }$. As is obvious from Table $\mathrm{I}$, the $\mathrm{pH}$ value of the mixture at $\tau_{\max }$ is lower than that of either component before mixing for A and B systems, but higher

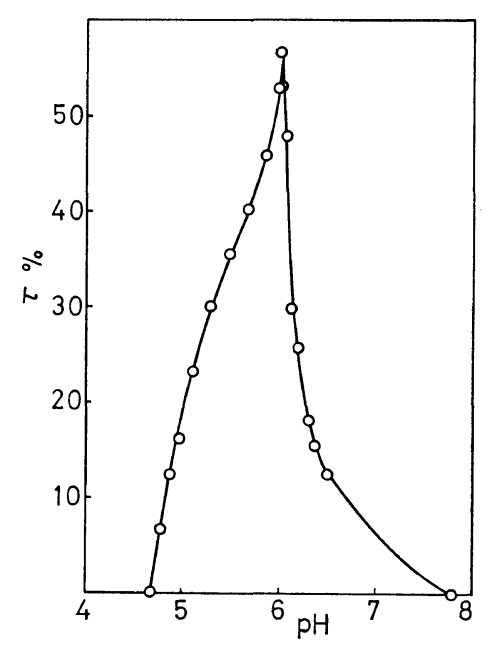

Figure 5. Relation between turbidity and $\mathrm{pH}$ when PEI aqueous solution is added to CMC solution; polymer concn, $4 \times 10^{-3} \%$.

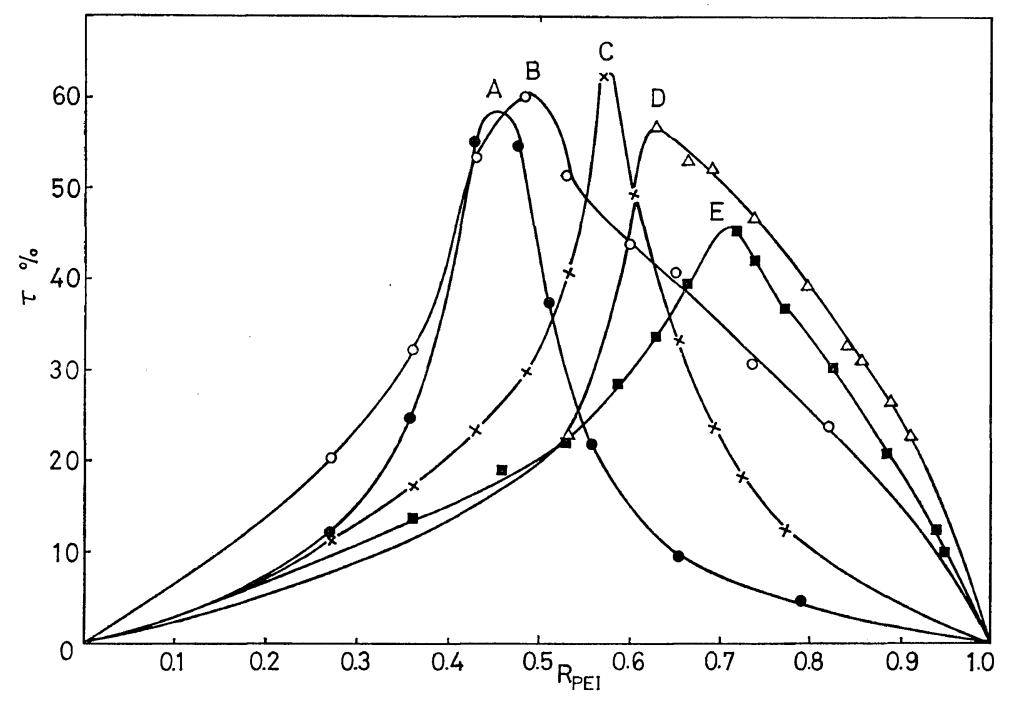

Figure 6. Turbidity plotted against mixing ratio $R_{\mathrm{PEI}}$ at different $\mathrm{pH}$ values: $\mathrm{pH}=3.35$ (A), 3.69 (B), 5.80 (C), 6.80 (D), 8.03 (E); polymer concn., $4 \times 10^{-3} \%$. 
Polyelectrolyte Complex from Carboxymethyl Cellulose and Poly(ethylenimine)

Table I. Change of $\mathrm{pH}$ after mixing of CMC and PEI solutions

\begin{tabular}{|c|c|c|c|c|c|c|}
\hline & \multicolumn{2}{|c|}{$\mathrm{pH}$ of Initial Solution } & \multirow{2}{*}{$\begin{array}{l}\mathrm{pH} \text { of Mixture } \\
\text { at } \tau_{\max }\end{array}$} & \multirow{2}{*}{$\begin{array}{l}\text { Change } \\
\text { of } \mathrm{pH}\end{array}$} & \multicolumn{2}{|c|}{$\alpha$ at $\tau_{\max }$} \\
\hline & $\mathrm{pH}_{\mathrm{CMC}}$ & $\mathrm{pH}_{\mathrm{PEI}}$ & & & $\alpha_{\mathrm{CMC}}$ & $\alpha_{\mathrm{PEI}}$ \\
\hline A & 3.43 & 3.47 & 5.35 & Decrease & 0.180 & 1.0 \\
\hline B & 3.82 & 3.76 & 3.69 & " & 0.367 & 1.0 \\
\hline $\mathrm{C}$ & 5.22 & 5.26 & 5.80 & Increase & 0.794 & 0.602 \\
\hline D & 6.30 & 6.40 & 6.80 & " & 1.0 & 0.397 \\
\hline $\mathrm{E}$ & 7.82 & 7.99 & 8.03 & " & 1.0 & 0.133 \\
\hline
\end{tabular}

for C, D, and E systems. Such a behavior may reflect the migration of protons accompanying the formation of a polyelectrolyte complex. When the degree of dissociation of PEI is sufficiently higher than that of CMC as in the cases of A and B, carboxyl groups are induced to dissociate by ionized PEI and protons are released from the carboxyl groups in CMC. Consequently the $\mathrm{pH}$ values of solutions become lower, and at the same time further ionic linkages are formed between CMC and PEI. On the contrary, protons are incorporated into PEI in the cases of $\mathrm{C}, \mathrm{D}$, and $\mathrm{E}$; this causes an increase in the $\mathrm{pH}$ of the mixtures. Reaction schemes in both cases are shown in the following:

Decrease in $\mathrm{pH}$ :

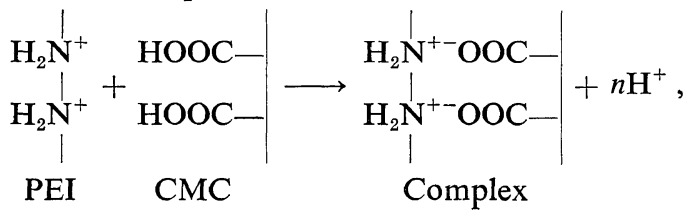

Increase in $\mathrm{pH}$ :<smiles>CCCN[N+](=O)[O-]</smiles>

The composition of the polyelectrolyte complex which appeared at the maximum point was regarded as the mixing ratio at $\tau_{\max }$. Because the maximum amount of polyelectrolyte complex seemed to be formed when the mixing ratio of polymers in a mixture is equal to the polymers composition in the complex. The experimental values of the polymer composition in the complexes given by the mixing ratio at $\tau_{\max }$, shown in Figure 6, are plotted against the $\mathrm{pH}$ values at $\tau_{\max }$ in curve a of Figure 7 . If the formation

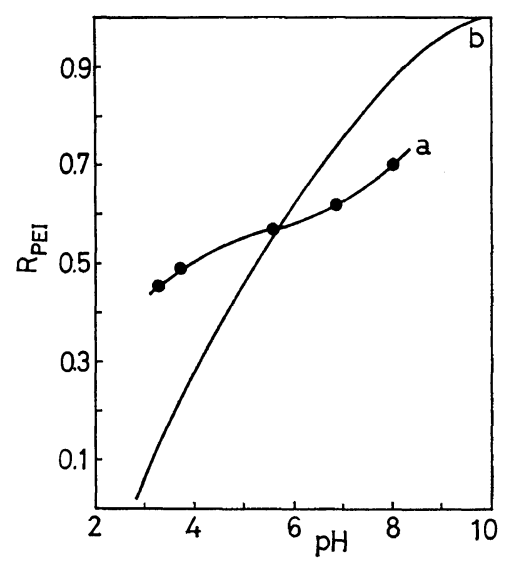

Figure 7. Dependence of $R_{\mathrm{PEI}}$ at maximum turbidity on $\mathrm{pH}$ : a, experimental curve for polyelectrolyte complex composed of PEI and CMC; $\mathrm{b}$, theoretical curve calculated from eq 4 for PEICMC polyelectrolyte complex.

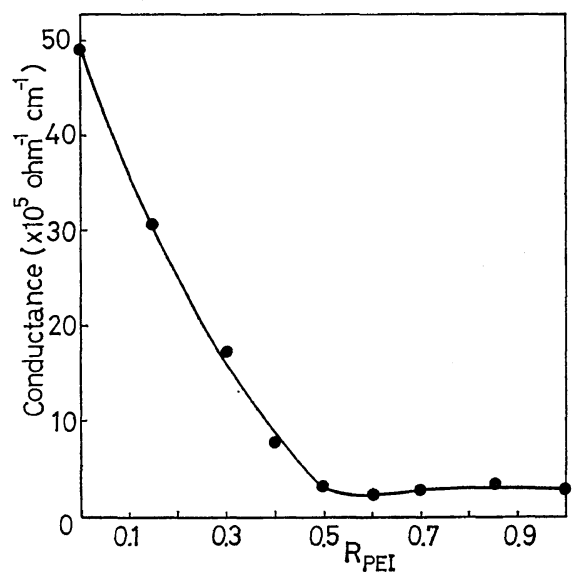

Figure 8. Conductance plotted against mixing ratio $R_{P E I}$ for polyelectrolyte complex solution prepared from $0.01-N \mathrm{CMC}$ and $0.01-N$ PEI aqueous solutions at $25 \pm 0.01^{\circ} \mathrm{C}$. 


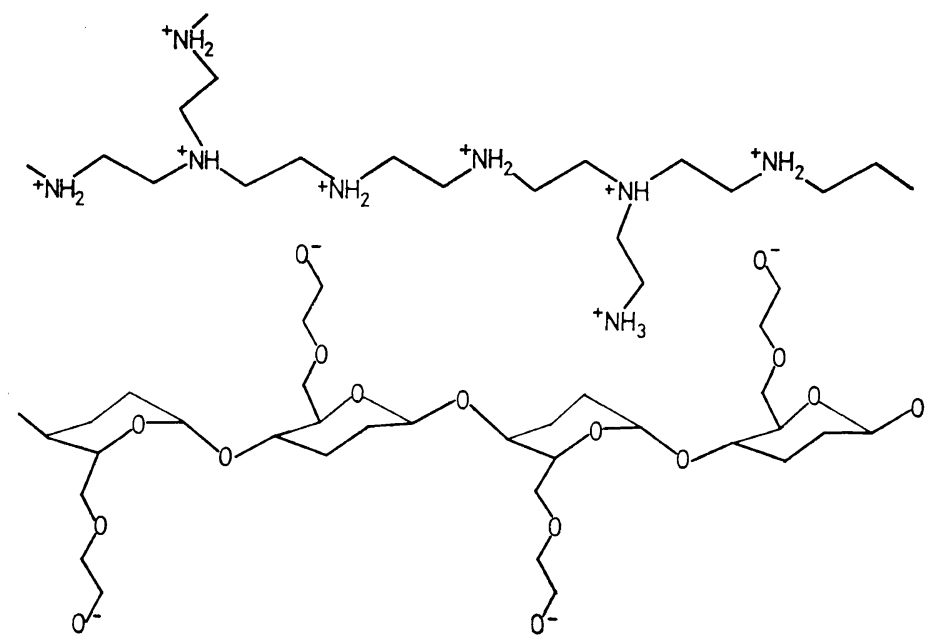

Figure 9. Extended conformations of PEI and CMC.

of a polyelectrolyte complex with CMC and PEI obeys the stoichiometrical reaction, then the following equation should hold:

\section{Hence}

$$
C_{\mathrm{CMC}} \alpha_{\mathrm{CMC}}=C_{\mathrm{PEI}} \alpha_{\mathrm{PEI}}
$$

$$
R_{\mathrm{PEI}}=\frac{C_{\mathrm{PEI}}}{C_{\mathrm{CMC}}+C_{\mathrm{PEI}}}=\frac{\alpha_{\mathrm{CMC}}}{\alpha_{\mathrm{CMC}}+\alpha_{\mathrm{PEI}}}
$$

Curve $b$ in Figure 7 represents the relation calculated from eq 4 by using the results given in Figure 3. Comparison of curve a with curve $b$ tells us that the formation of a polyelectrolyte complex for the present system does not obey the stoichiometry and is rather close to the reaction between strong polyelectrolytes. We may expect that there are no ionized groups at the $R_{\mathrm{PEI}}$ where curve a crosses curve b. To examine whether ionized groups are present or not at this $R_{\mathrm{PEI}}$, the conductance of the solution was next measured. PEI and CMC were separately dissolved in conductance water, and then the PEI solution was added to the CMC solution. In Figure 8, the conductance is plotted against $R_{\mathrm{PEI}}$. It was difficult to obtain the precise minimum point because of the low conductance of the PEI solution, but the conductance never became zero. This fact should support the presence of ionized groups on PEI chains. Such a result is different from that of Michaels ${ }^{4}$ for complex formation with strong vinyl polyelectrolytes, in which the reaction was concluded to be complete from the fact that the conductance was zero at the minimum point.

We suggest that such behaviors for our polyelectrolyte complex may be attributed to the less flexible nature of CMC chains, high density of ionizable groups in PEI, and/or branching in PEI. The fully extended conformations of CMC and PEI are shown in Figure 9. The distance between neighboring ionizable groups of PEI is considerably shorter than that of CMC, which is suppposed to leave free positive charges in the complex. Consequently the remarkable deviation from the experimental curve shown in Figure 7 may be explained by the following two reasons; first, release or incorporation of protons by a polyelectrolyte is induced by the partner polyelectrolyte having the opposite charge; second, geometrical hindrance in ionic linkages may leave free charges of PEI particularly in the region of the acidic side.

Waynerman, et al., ${ }^{10}$ have reported that the complex formation between arginic acid, a weak and rigid polyanion, and gelatin, a weak polycation, depends on $\mathrm{pH}$ and is stoichiometric. Further research seem to be necessary. But we should emphasize the effect of chain conformations of complex formation. Our conclusion for the present system is that the complex formation is not stoichiometric because of conformational effects of both components and of the induced ionization of undissociated residues by oppositely charged residues. 


\section{Polyelectrolyte Complex from Carboxymethyl Cellulose and Poly(ethylenimine)}

\section{REFERENCES}

1. A. Nakajima and H. Sato, Biopolymers, 11, 1345 (1972).

2. H. Terayama, Kagaku no Kenkyu, 1, 75 (1948).

3. H. Terayama, J. Polym. Sci., 8, 243 (1952).

4. A. S. Michaels, L. Mir, and N.S. Schneider, J. Polym. Sci., 69, 1447 (1965).

5. M. Hosono, S. Sugii, O. Kusudo, and W. Tsuji, Report of the Poval Committee, Kyoto, 61, 79 (1972).

6. R. M. Fuoss and H. Sadek, Science, 110, 552
(1949).

7. M. Hosono, O. Kusudo, and W. Tsuji, Report of the Poval Committee, Kyoto, 57, 99 (1970).

8. G. D. Jones A. Langsjoen, M. M. C. Neumann, and J. Zomlefer, J. Org. Chem., 9, 125 (1944).

9. H. G. Bungenberg de Jong, "Colloid Science," Vol. II, H. R. Kruyt, Ed., Elsevier, Amsterdam, 1949.

10. E. S. Wajnerman, W. Ja. Grinberg, and W. B. Tolstogusow, Kolloid-Z. Z. Polym., 250, 945 (1972). 